\title{
Epigallocatechin gallate (EGCG) suppresses $\beta$-amyloid-induced neurotoxicity through inhibiting c-Abl/FE65 nuclear translocation and GSK3 $\beta$ activation
}

\author{
Chih-Li Lin ${ }^{\mathrm{a}}$, Ta-Fu Chen ${ }^{\mathrm{b}}$, Ming-Jang Chiu ${ }^{\mathrm{b}}$, Tzong-Der Way ${ }^{\mathrm{a}}$, Jen-Kun Lin ${ }^{\mathrm{a}, *}$ \\ a Institute of Biochemistry and Molecular Biology, College of Medicine, National Taiwan University, Taipei, Taiwan \\ ${ }^{\mathrm{b}}$ Department of Neurology, National Taiwan University Hospital, Taipei, Taiwan \\ Received 14 December 2006; received in revised form 1 May 2007; accepted 11 May 2007 \\ Available online 22 June 2007
}

\begin{abstract}
Alzheimer's disease (AD) is the most common neurodegenerative disease and is caused by an accumulation of A $\beta$ plaque deposits in the brains. Evidence is increasing that green tea flavonoids can protect cells from A $\beta$-mediated neurotoxicity. However, the underlying mechanism remains unclear. Here, we used a human neuronal cell line MC65 conditional expression of an amyloid precursor protein fragment (APPC99) to investigate the protection mechanism of epigallocatechin gallate (EGCG), the main constituent of green tea. We demonstrated that treatment with EGCG reduced the A $\beta$ levels by enhancing endogenous APP nonamyloidogenic proteolytic processing. Furthermore, EGCG also decreased nuclear translocation of c-Abl and blocked APP-C99-dependent GSK3 $\beta$ activation, and these inhibitory effects occurred through the interruption of $\mathrm{c}-\mathrm{Abl} / \mathrm{Fe} 65$ interaction. Our results indicated that the neuroprotective action of EGCG may take place through some mechanisms other than the promotion of APP nonamyloidogenic proteolysis, as was reported previously.
\end{abstract}

(C) 2007 Elsevier Inc. All rights reserved.

Keywords: (-)-Epigallocatechin gallate; $\beta$-amyloid; c-Abl; FE65; Glycogen synthase kinase $3 \beta$

\section{Introduction}

Alzheimer's disease (AD) is currently the most common type of neurodegenerative dementia. It is generally accepted that the deposition of cerebral $\beta$-amyloid $(A \beta)$ peptides is the primary cause of AD pathology (Drachman, 2006). A $\beta$ originates from the proteolytic processing of a transmembrane glycoprotein called amyloid precursor protein (APP) via the coordination of $\alpha-, \beta$-, and $\gamma$-secretases. Cleavage of APP by $\alpha$-secretase results in the formation of the non-harmful soluble $\alpha$-APP (sAPP $\alpha$ ). However, the A $\beta$-containing C-terminal fragment (CTF), known as $\beta-\mathrm{CTF}$, is produced by $\beta$-secretase

\footnotetext{
* Corresponding author at: Institute of Biochemistry and Molecular Biology, National Taiwan University College of Medicine, Room 947, No. 1, Sec. 1, Jen-Ai Rd., Taipei 100, Taiwan. Tel.: +886 22312 3456x2213; fax: +886223918944.

E-mail address: jklin@ha.mc.ntu.edu.tw (J.-K. Lin).
}

cleavage (Sinha and Lieberburg, 1999) and subsequent cleavage by $\gamma$-secretase results in the formation of the insoluble amyloidogenic $A \beta$. It has been proposed that the deposit of the dysregulated $A \beta$ forms the plaque core and subsequently induces GSK3 $\beta$ activation, tau aggregation, abnormal tau hyperphosphorylation, neuronal attrition, and then clinical dementia (Iqbal and Grundke-Iqbal, 2006). Genetic analysis showed that some AD cases are familial with autosomal dominant inheritance of mutation in genes, including APP and preseniline locus (Selkoe, 2004). The combination of genetic and biochemical data suggests that $A \beta$ deposition is the primary event in $\mathrm{AD}$ and forms the basis of the amyloid cascade hypothesis in AD pathogenesis (Hardy and Higgins, 1992).

The actual mechanisms involved in $\mathrm{A} \beta$-mediated neurotoxicity are not very clear, but more and more evidence suggests that oxidative stress may play a key role. Beyond the direct effects of $A \beta$-mediated neurotoxicity, oxidative dam- 
age was also revealed in the AD brain (Smith et al., 1996). The mammalian brain is especially susceptible to oxidative stress (Floyd and Hensley, 2002), and AD brains exhibited evidence of reactive oxygen species (ROS)-mediated injury (Blass, 2002). Previous studies had implied that A $\beta$ toxicity and aggregation involve oxidative stress resulting from the accumulation of ROS (Miranda et al., 2000). A $\beta$ neurotoxicity was also shown to be attenuated by numerous antioxidants (Dai et al., 2006). In recently years, epidemiological studies indicated that nutritional antioxidants can reduce neuronal death (Esposito et al., 2002). There is also increasing interest for the role of tea (Camellia sinensis) in anti-dementia activity. Catechins and polyphenols, the main bioactive chemicals in tea and related compounds, can scavenge ROS and exert protective and neurorescue effects against amyloid neurotoxicity (Mandel et al., 2006). In particular, the neuroprotective effects of (-)-epigallocatechin-3-gallate (EGCG), the major polyphenol isolated from green tea, have been investigated in several studies (Mandel et al., 2003). In some in vitro studies, EGCG was shown to prevent $A \beta$ peptide-treated neuronal cell death (Choi et al., 2001). Moreover, EGCG was demonstrated to reduce $\mathrm{A} \beta$ levels both in vitro and in vivo by promoting APP nonamyloidogenic cleavage (Levites et al., 2003). Thus, emerging evidence suggests that the neuroprotective action of EGCG comes from antioxidant activity and also from the ability to alter signaling pathways in the neuronal damage process. However, some results showed that direct co-treatment of neuronal cells with exogenous $A \beta$ and EGCG demonstrated significant neuroprotective effects (Choi et al., 2001), suggesting that EGCG or other antioxidant compounds may exert their protective effects through mechanisms other than promoting APP nonamyloidogenic proteolysis.

$\mathrm{c}-\mathrm{Abl}$ is a cytoplasmic nonreceptor tyrosine kinase involved in the development of the nervous system (Van Etten, 1999) and also implicated in the regulation of cell apoptosis (Wang, 2005). Treatment of neuronal cells with A $\beta$ fibrils induced an increased neurotoxicity associated with c-Abl nuclear translocation (Alvarez et al., 2004). Based on this, $A \beta$ cytotoxicity is considered a crucial stress factor causing neuronal cells to enter cell death in the mammalian AD brain. Additionally, it was shown that FE65, a brain-enriched adaptor protein, can be phosphorylated by c-Abl and then co-translocated to the nucleus to regulate gene transcription (Perkinton et al., 2004). The neuron-specific adaptor protein FE65 binding with phosphorylated APP intracellular domain (AICD) at $\mathrm{Thr}^{668}$ could induce nuclear translocation and affect the sequent neurotoxicity, which was possibly mediated through the induction of glycogen synthase kinase $3 \beta$ (GSK3 $\beta$ ) and tau phosphorylation by enhancing the formation of a ternary complex with Fe65 and CP2 transcription factor (Nakaya and Suzuki, 2006).

In the present study, we used a human neuronal cell line MC65 stably transfected with APP-C99 gene. Conditional expression of APP-C99 was performed using a tetracyclineresponsive promoter system, and the activity of this promoter is tightly regulated by the presence or absence of the tetracycline in the culture medium. In the presence of tetracycline the promoter activity is repressed, and by removal of tetracycline from the media overexpression APP-C99 transgene can be induced (Supplemental Fig. 1). Therefore, the MC65 cell is a convenient tool to help us understand the mechanisms by which intraneuronal $A \beta$ accumulates and induces toxicity. We demonstrated that EGCG treatment of MC65 cells overexpressed with APP-C99 leads to a significant reduction in cell death. The treatment with EGCG reduced the A $\beta$ levels by enhancing APP nonamyloidogenic processing, and also decreased nuclear subcellular localization of c-Abl, which was independent of APP processing. A similar result was also revealed in the experiment involving a simple neuroblastoma cell line SK-N-MC co-treated with A $\beta$ and EGCG. These findings provide a further mechanism in blocking A $\beta$-induced neurotoxicity by the EGCG treatment.

\section{Materials and methods}

\subsection{Reagents}

Green tea-derived flavonoid (-)-epigallocatechin3-gallate (EGCG), (-)-epigallocatechin (EGC), (-)-epicatechin-3-O-gallate (ECG), (-)-epigallocatechin (EC) and catechin (C) were purchased from Sigma (München, Germany). Antibodies against A, APP, and phosphoThr ${ }^{668}$-APP were obtained from Chemicon (Temecula, CA), and those against c-Abl, TFIIB, and $\beta$-actin were purchased from Santa Cruz Biotechnology (Santa Cruz, CA). GSK3 $\beta$ and phosphoTyr ${ }^{216}$-GSK3 $\beta$ antibody were from Cell Signaling Technology, Inc. (Beverly, MA). The anti-FE65 antibody was from Upstate Biotechnology, Inc. (Lake Placid, NY). Gleevec (Imatinib mesylate), obtained from the Novartis Company, was a relatively well-tolerated selective inhibitor of tyrosine kinases used as a positive control in the study.

\subsection{Cell cultures}

The MC65 cell line was a gift from the Dr. George Martin's laboratory, University of Washington (Seattle, WA, USA). It originated from the human neuroblastoma cell line SK-N-MC transfected by vector p10C99, which was engineered to express a partial APP protein APP-C99 (amino-17 residues + carboxyl-99 residues) under control of the tetracycline-controlled transactivator as described previously (Sopher et al., 1994). Cells were routinely cultured in Dulbecco's modified Eagle's medium (DMEM), supplemented with $4.5 \mathrm{mg} / \mathrm{ml} \mathrm{D}$-glucose, penicillin $(50 \mathrm{IU} / \mathrm{ml})$, streptomycin $(50 \mu \mathrm{g} / \mathrm{ml})$, non-essential amino acids, sodium pyruvate $(1 \mathrm{mM})$, and $10 \%$ heat-inactivated fetal bovine serum. At least $1 \mu \mathrm{g} / \mathrm{ml}$ tetracycline was added to inhibit amyloid protein production before the experiment. 


\subsection{Cytotoxicity assays}

For cytotoxic experiments, cells were cultured in 24well culture dishes, seeded at $2 \times 10^{5}$ cells per well, and maintained in a logarithmic growth phase for 3 days, following growth in a tetracycline-free medium to stimulate production of the amyloid protein. Then, the cells were treated with EGCG or Gleevec with or without tetracycline in the culture medium. After incubation, cell viability was measured by the modified 3-[4,5-dimethylthiazol-2-yl]2,5-diphenyltetrazolium bromide (MTT) assay method using DMEM medium without phenol red. Absorbance of the converted dye was measured at a wavelength of $550 \mathrm{~nm}$ with a microplate reader (Mosmann, 1983).

\subsection{Immumoprecipitation}

The total cell lysate was centrifuged at $12,000 \mathrm{rpm}$ for $20 \mathrm{~min}$, and the aliquot of supernatant (500 $\mu \mathrm{g}$ total protein) was then incubated with antibodies in immunoprecipitation buffer ( $\mathrm{pH} 7.4)$ and gently rocked overnight at $4{ }^{\circ} \mathrm{C}$. The immunocomplexes were adsorbed to protein A-agarose beads for $2 \mathrm{~h}$ at $4{ }^{\circ} \mathrm{C}$ during gentle agitation and subsequently collected by centrifugation at $14,000 \mathrm{rpm}$ for $30 \mathrm{~s}$ at $4{ }^{\circ} \mathrm{C}$. Beads were then washed thrice with ice-cold PBS, incubated for $10 \mathrm{~min}$ at $95^{\circ} \mathrm{C}$ with $20 \mu \mathrm{l}$ electrophoresis buffer, and the complete supernatant was used for Western blot analysis.

\subsection{ELISA of A $\beta$ content}

Samples were placed in ice-cold lysis buffer, sonicated on ice for $3 \mathrm{~min}$, allowed to stand for $15 \mathrm{~min}$ at $4{ }^{\circ} \mathrm{C}$, and centrifuged at $15,000 \mathrm{rpm}$ for $15 \mathrm{~min}$. $A \beta_{1-40,42}$ species were detected by acid extraction of brain homogenates in $5 \mathrm{M}$ guanidine buffer, followed by a 1:10 dilution in lysis buffer. In the measurements of brain $A \beta$, frozen hemibrains were sequentially extracted in a two-step extraction (sonication in 2\% SDS and 70\% formic acid). After sonication the samples were centrifuged at $100,000 \times g$ for $1 \mathrm{~h}$ at $4{ }^{\circ} \mathrm{C}$. The supernatant was recovered, and the pellet was sonicated with the next solution. $A \beta_{1-40}$ or $A \beta_{1-42}$ was separately quantified using the specific ELISA kits (Biosources, Camarillo CA) in accordance with the manufacturer's instructions.

\subsection{Western blot analysis}

Cells were harvested and homogenized in a lysis buffer (50 mM Tris- $\mathrm{HCl}$, pH 8.0, $5 \mathrm{mM}$ EDTA, $150 \mathrm{mM} \mathrm{NaCl}$, $0.5 \%$ Nonidet P- $40,0.5 \mathrm{mM}$ phenylmethylsulfonyl fluoride, and $0.5 \mathrm{mM}$ dithiothreitol) for $30 \mathrm{~min}$ at $4{ }^{\circ} \mathrm{C}$. Equal amounts of total cellular proteins $(50 \mu \mathrm{g})$ were resolved by SDS-PAGE, transferred onto polyvinylidene difluoride membranes (Amersham Biosciences), and then probed with primary antibody followed by secondary antibody conjugated with horseradish peroxidase. The immunocomplexes were visualized with enhanced chemiluminescence kits (Amersham Biosciences).

\subsection{RNAi suppression of $\mathrm{c}-\mathrm{Abl}$}

The c-Abl siRNA gene silencer (human) dsRNA was obtained from Santa Cruz Biotechnology (sc-29843). Neuroblastoma cells were transfected with dsRNAs using siRNA Transfection Reagent (Santa Cruz) and incubated for $6 \mathrm{~h}$. Afterward, the cells were analyzed by immunoblot for cAbl expression or MTT assay for cell viability determination.

\subsection{Immunofluorescence}

MC65 cells were cultured on coated slides and treated for $72 \mathrm{~h}$. After treatment cells were fixed with $2 \%$ buffered paraformaldehyde, permeabilized in $0.25 \%$ Triton X-100 (Sigma-Aldrich) for $5 \mathrm{~min}$ at $4{ }^{\circ} \mathrm{C}$, and then incubated with anti-c-Abl and fluorescent dye 4',6-diamidino-2phenylindole (DAPI). The slides were then incubated with an FITC labeled second antibody (Santa Cruz) depending on the origin of the primary antibody. Slides were viewed using the Leica TCS SP2 Spectral Confocal System and MetaMorph, V 4.5 software.

\subsection{Mice}

The heterozygous $\operatorname{Tg} 2576$ amyloid precursor protein mouse model was purchased from Taconic (Germantown, NY). We then backcrossed Tg2576 with C57BL/6 with Taconic's permission. The mice of the first generation were screened for the presence of APPsw and a-TTP genes by PCR analysis of tail DNA as described before (Hsiao et al., 1996). Thirty-five $\operatorname{Tg}(+)$ mice and seventeen $\operatorname{Tg}(-)$ mice were used in the study. Half the 8-month-old $\operatorname{Tg}(+)$ mice were orally administered with EGCG $(20 \mathrm{mg} / \mathrm{kg})$ daily for 4 months. Then all the mice were sacrificed at 12 months of age and analyzed for $A \beta$ levels and $A \beta$ load in the brain according with an Elisa kit and Congo red staining. Animals were housed and maintained at the College of Medicine Animal Facility of National Taiwan University, and all experiments were in compliance with protocols approved by the National Taiwan University Institutional Animal Care and Use Committee.

\subsection{Statistical analysis}

Data are presented as means \pm S.E. Statistical significance was set at $p<0.05$. Statistically significant differences were determined by the analysis of variance in SPSS statistical software (SPSS, Inc., Chicago, IL). 


\section{Results}

\subsection{EGCG treatment against neurotoxicity in APP-C99-overexpressed cultured cells}

In agreement with data obtained in previous APPoverexpressed studies, our first results reveal that 48-96 h of APP-C99 overexpression caused a progressive decrease in MTT reduction (Fig. 1A). This demonstrated that MC65 cells subjected to conditional expression of APPC99 could show neuronal cell toxicity. To evaluate the neuronal protection effect of tea polyphenolic substances, we used five of the major components present in green tea - (-)-epigallocatechin-3-gallate (EGCG), (-)-epigallocatechin (EGC), (-)-epicatechin-3-O-gallate
(ECG), (-)-epigallocatechin (EC), and catechin - to co-treat MC65 cells. The toxicity effect was shown to be reduced by a treatment with gallate forms of tea polyphenols like EGCG and ECG. In contrast, non-gallate forms like catechin, EC and EGC displayed fewer protective effects against APP-C99-induced neurotoxicity (Fig. 1B). Among these polyphenols, EGCG is the most potent compound in our assay $\left(\mathrm{EC}_{50}=6.75 \mu \mathrm{M}\right)$. These protective effects occur in a dose-dependent manner and are significant at 5-10 $\mu \mathrm{M}$ and maximal at $20 \mu \mathrm{M}$ (Fig. $1 \mathrm{C}$ and D).

Recent evidence had reported EGCG markedly promotes cleavage of APP with elevated $\alpha$-secretase activity in vitro and in vivo (Rezai-Zadeh et al., 2005). Based on these reports, we validated the APP proteolytic processing using the ELISA method to measure $A \beta$ levels. As shown in Fig. $2 \mathrm{~A}$, we
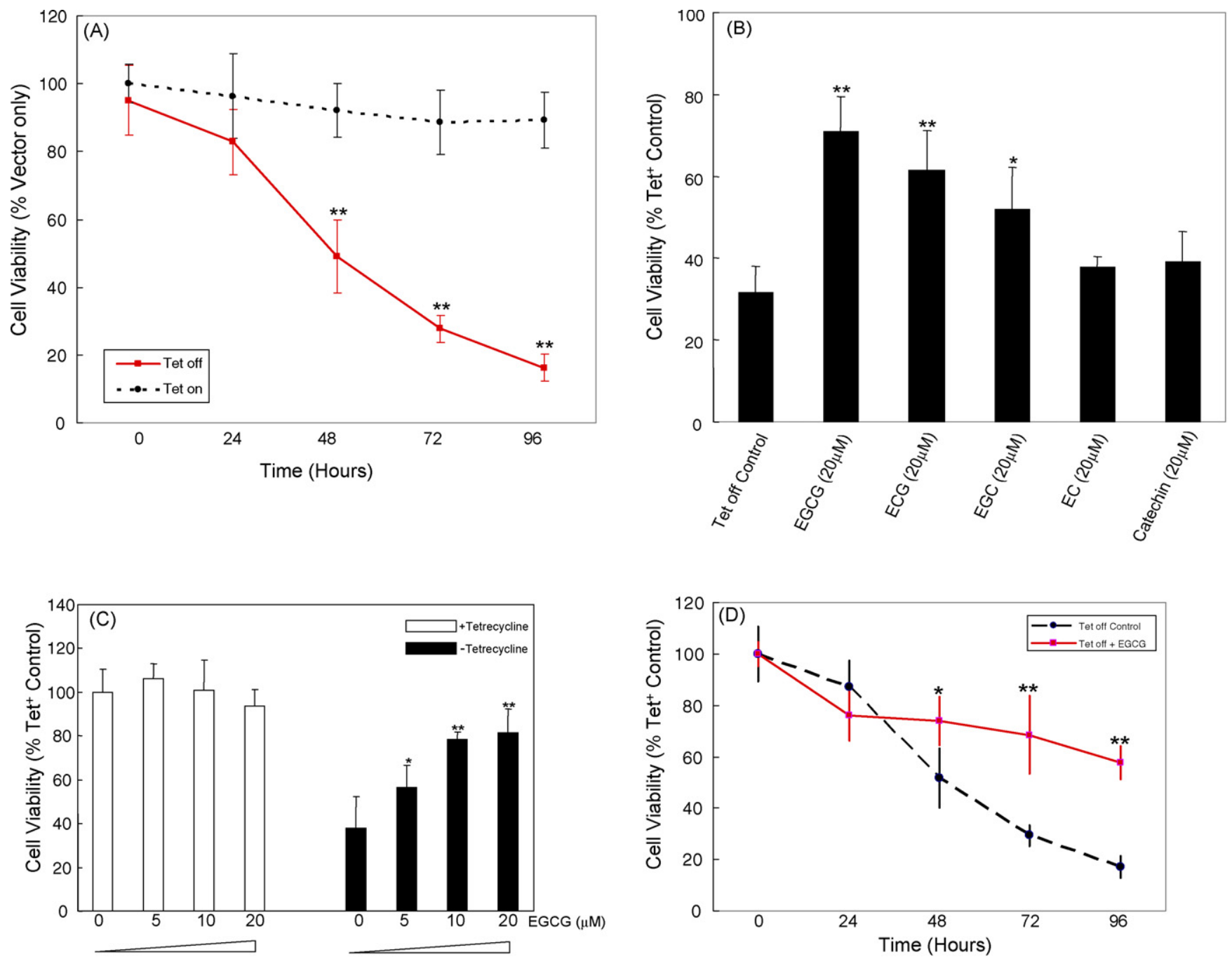

Fig. 1. EGCG treatment against neurotoxicity in APP-C99-overexpressed cultured MC65 cells. (A) APP-C99 overexpression was induced in MC65 cells by removing the tetracycline medium and a significant neurotoxicity was demonstrated after 48-96h. (B) Effects of (-)-epigallocatechin-3-gallate (EGCG), (-)-epigallocatechin (EGC), (-)-epicatechin-3-O-gallate (ECG), (-)-epigallocatechin (EC), and catechin against APP-C99-induced toxicity at 48 h after removing the tetracycline medium. Among these polyphenols, EGCG is the most potent compound with $\mathrm{EC}_{50}$ at $6.75 \mu \mathrm{M}$. (C) EGCG protective effect occurred in a dose-dependent manner and was significant at 5-20 $\mu \mathrm{M}$, (D) and the protective effect could be sustained for $96 \mathrm{~h}$. Bars depict mean \pm S.E. of at least three independent experiments. ' $*$ ' and '**' represent a statistically significant difference from a non-tetracycline-treated control group, $p<0.05$ and $p<0.01$, respectively. 

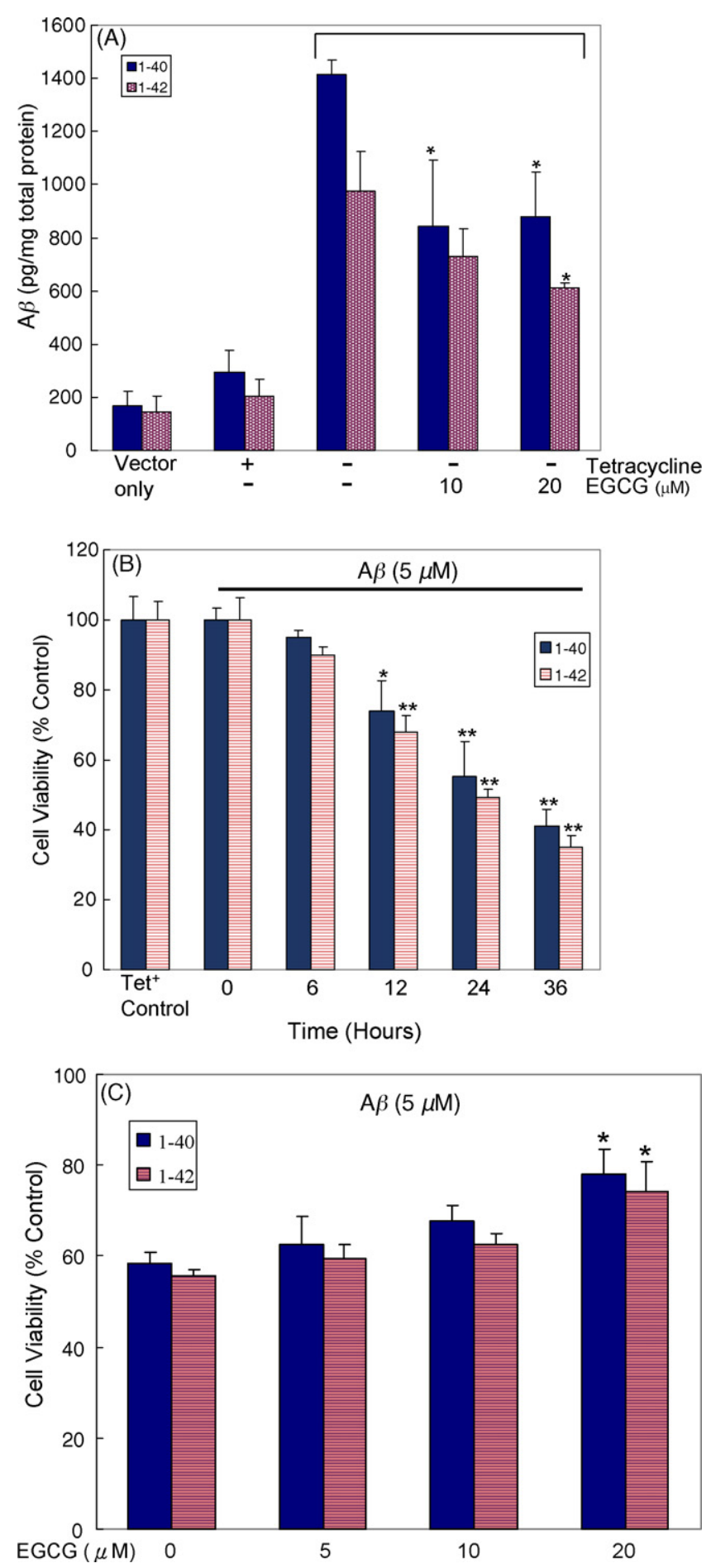

Fig. 2. EGCG inhibits $A \beta$ generation and exogenous $A \beta$-induced neuronal death. (A) EGCG reduced both $A \beta_{1-40}$ and $A \beta_{1-42} A \beta$ intracellular peptide generation in MC65 cells according to analysis by ELISA method. Cells treated with EGCG reduced A $\beta$ generation by $20-30 \%$ at $20 \mu \mathrm{M}$ compared with untreated cells. (B) The treatment of human SK-N-MC neuroblastoma cells with exogenous $A \beta_{1-40}$ and $A \beta_{1-42}$ fibrils $(5 \mu \mathrm{M})$ for $12-36 \mathrm{~h}$ promoted cell death significantly. (C) The EGCG treatments of SK-N-MC cells were also able to decrease MTT reduction induced by exogenous supplement of $A \beta_{1-40}$ and $A \beta_{1-42}$ fibrils for $24 \mathrm{~h}$ in a dose-dependent manner, and were significant at $20 \mu \mathrm{M}$. Bars depict mean \pm S.E. of at least three experiments. ' $*$ ' and ' $* *$ ' represent statistically significant differences from a non-tetracycline-treated control group, $p<0.05$ and $p<0.01$, respectively. found that EGCG could reduce intracellular $A \beta$ generation (both $A \beta_{1-40}$ and $A \beta_{1-42}$ peptides) in a dose-dependent manner. MC65 cells treated with EGCG reduced $A \beta$ generation by $20-30 \%$ at $20 \mu \mathrm{M}$ compared with untreated cells. In addition to the reduced $A \beta$ levels, EGCG also showed a dose-dependent effect on soluble APP (sAPP) release in culture medium (Supplemental Fig. 2). These data are consistent with those previously reported showing that EGCG can enhance the activity of metalloproteinase degrading $A \beta$ peptides (Levites et al., 2003) and elicit $\alpha$-secretase activity (Rezai-Zadeh et al., 2005).

\subsection{EGCG inhibits exogenous $A \beta$-induced neuronal death}

To study the relationship between $\mathrm{A} \beta$-induced neurotoxicity and EGCG protective effects, we evaluated the cell viability of neuronal cells exposed to exogenous $A \beta$ fibrils co-treated with EGCG. Compared to endogenous $A \beta$ induced by overexpression of APP-C99, treatment of human SK-NMC neuroblastoma cells to exogenous $A \beta_{1-40}$ and $A \beta_{1-42}$ fibrils for $24-48 \mathrm{~h}$ promoted cell death significantly (Fig. 2B). The EGCG treatments were able to decrease MTT reduction induced by exogenous supplement $A \beta_{1-40}$ and $A \beta_{1-42}$ fibrils for $24 \mathrm{~h}$ in a dose-dependent manner (Fig. 2C). These data suggested that EGCG is able to protect cultured neuronal cells against both APP-C99 and A $\beta$-induced toxicity. Intriguingly, EGCG significantly protects SK-N-MC cells from exogenous $\mathrm{A} \beta$ damage, indicating it may enhance cell survival through a mechanism other than the inhibition of $A \beta$ synthesis. These results give rise to the idea that although EGCG inhibits $A \beta_{1-40}$ and $A \beta_{1-42}$ generation from APP-C99 overexpressed MC65 cells, some other underlying mechanisms allow EGCG to prevent neuronal cell death. It is now known that the main effects of EGCG seem to be related to its free radical scavenging properties, raising the possibility that EGCG-mediated inhibition of $A \beta$ toxicity may also be accomplished via blockade of $A \beta$-induced ROS accumulation. To address this possibility, we evaluated the ROS levels in a cell line model. Results show that treatment with EGCG at a dose range was reported to block ROS accumulation in MC65 cells (Supplemental Fig. 3).

\subsection{The overexpression of APP-C99 increases $c-A b l$ translocation to nuclei}

The nuclear pool of $\mathrm{c}-\mathrm{Abl}$ has been implicated in neuronal death. In addition, $A \beta$ fibrils and ROS have also been demonstrated to activate neuronal c-Abl (Alvarez et al., 2004). To evaluate this point, we analyzed the subcellular distribution of c-Abl protein in APP-C99-overexpressed neuronal cells. In Fig. 3A, we observe that c-Abl expression dominates the cytoplasm of control MC65 cells. Induction of APP-C99 overexpression in neuronal cells enhanced a significant increase in c-Abl immunofluorescence in nuclei at $72 \mathrm{~h}$. Similarly, the c-Abl protein levels were elevated in the 
(A)
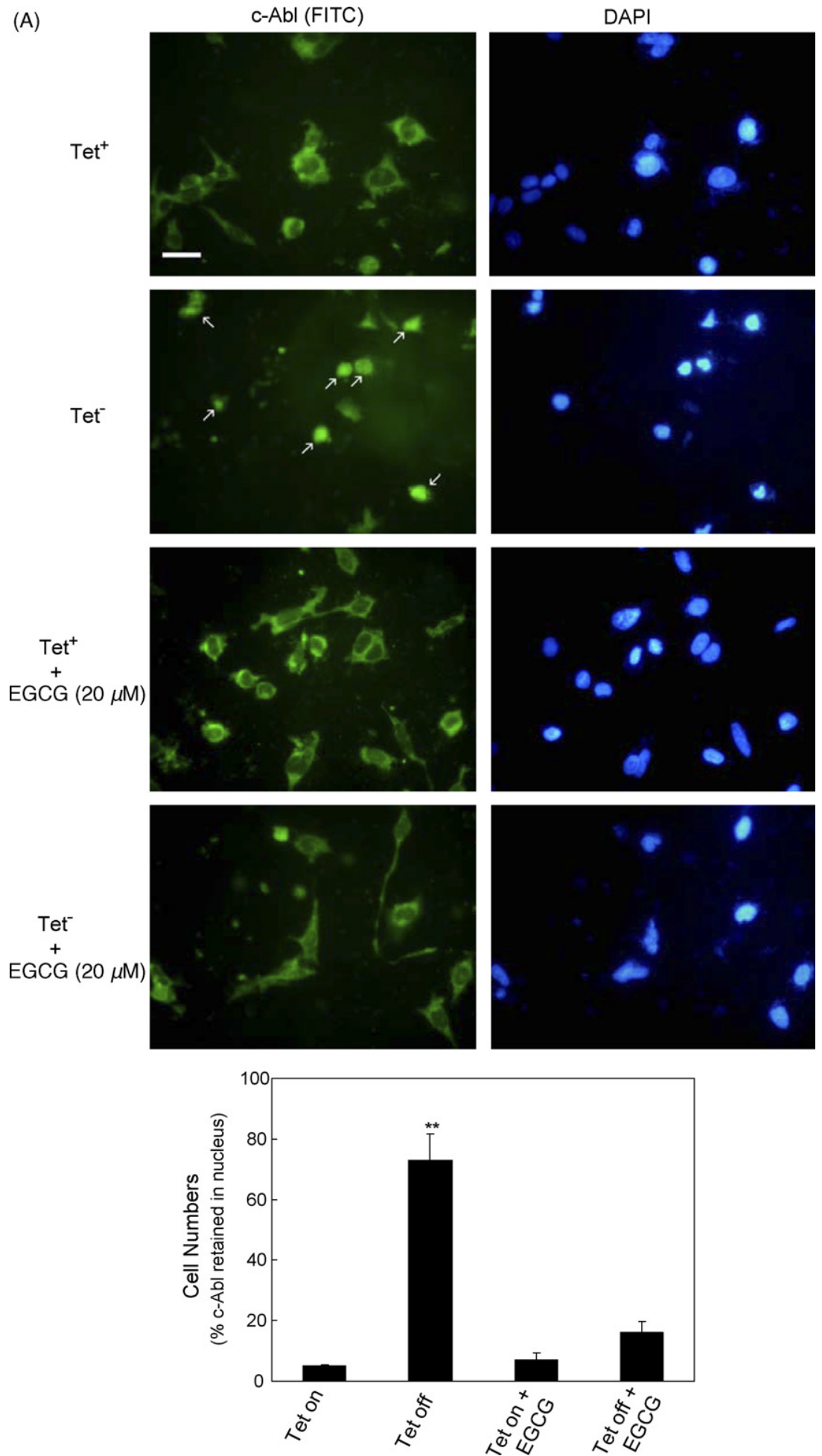

Fig. 3. The overexpression of APP-C99 increases nuclear c-Abl translocation. (A) Immunofluorescence images showed that neuronal cells in which APP-C99 overexpression was induced significantly increased the c-Abl expression in the nuclei at $72 \mathrm{~h}$. 4',6-Diamidino-2-phenylindole stained nuclei are detected in the images on the right. It was also shown that EGCG $(20 \mu \mathrm{M})$ treatment inhibits the nuclear translocation of c-Abl when APP-C99 is overexpressed in MC65 cells. Arrows indicated the c-Abl nuclear translocation of MC65 cells. (B) Similarly, the results of subcellular fractionation of c-Abl by Western blotting confirm the immunofluorescence results. c-Abl was significant decreased in the nuclear fraction compared to the non-EGCG treated control. (C) Treatment with Gleevec $(10 \mu \mathrm{M})$, a c-Abl kinase inhibitor, significantly decreased in APP-C99-induced cell death. Scale bar is $10 \mu \mathrm{m}$. '*' represents a statistically significant difference, $p<0.05$. 

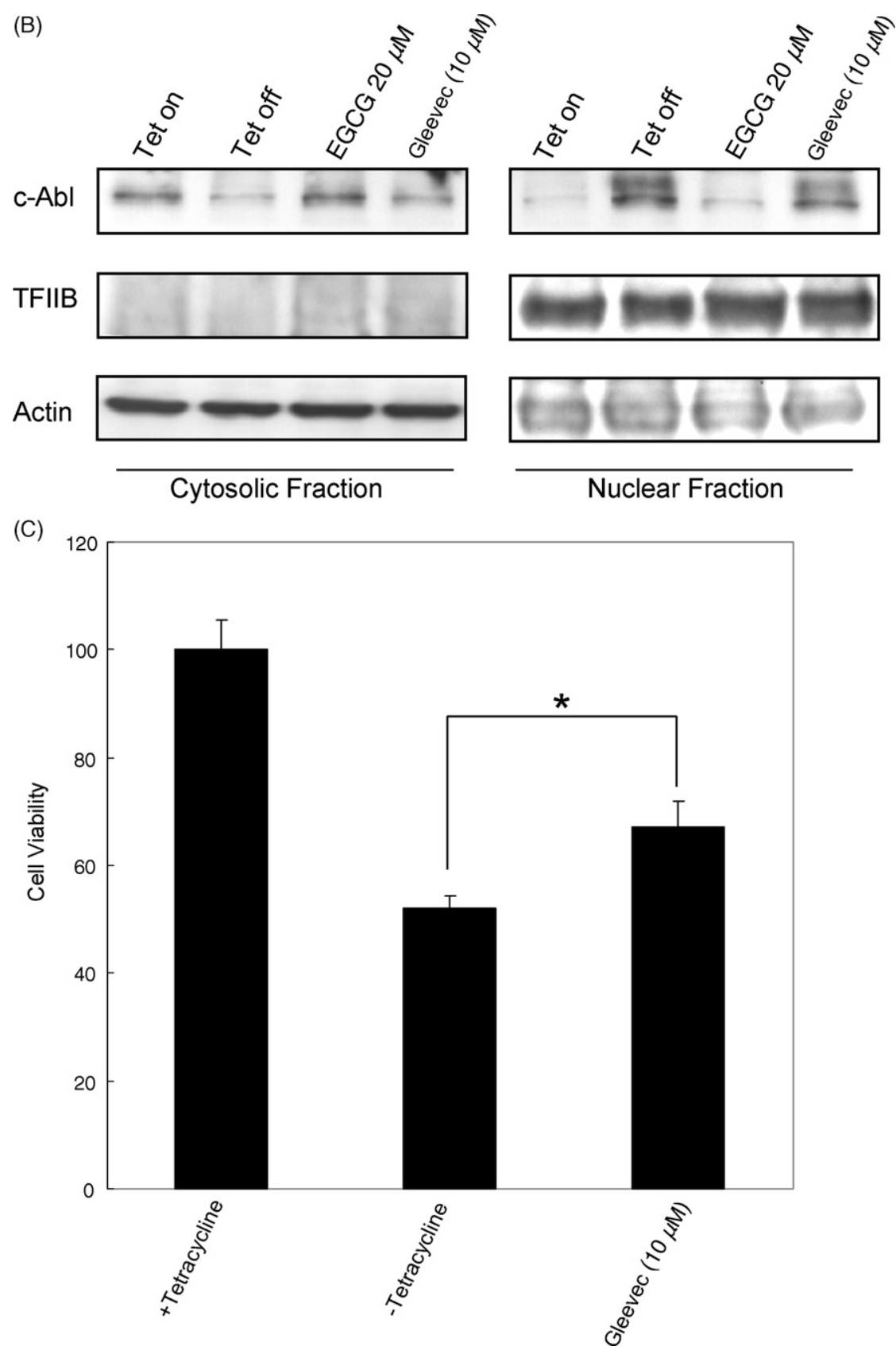

Fig. 3. (Continued).

nuclear fraction of those neuronal cells induced to APP-C99 overexpression by Western blotting and immunofluorescence analysis (Fig. 3B). In order to identify possible mechanisms involved in APP-C99-induced neurotoxicity, we next investigated the involvement of c-Abl nuclear translocation by EGCG treatments. As expected, our data also showed that EGCG $(20 \mu \mathrm{M})$ treatment inhibits the nuclear translocation of c-Abl under APP-C99 overexpression conditions in MC65 cells. These results support the idea that c-Abl may be involved in the neuronal cell death induced by APP overproduction. Thus, to validate the essential role of c-Abl, we used a clinical drug Gleevec, a well-known c-Abl kinase inhibitor reported to inhibit c-Abl-induced apoptosis in neuronal cells (Alvarez et al., 2004). Not surprisingly, the treatment with Gleevec $(10 \mu \mathrm{M})$ decreased cell death (Fig. 3C) but not cAbl nuclear translocation. These results indicated that the EGCG-inhibited c-Abl nuclear translocation is critical to APP-C99-induced neurotoxicity.

\subsection{Inhibition of $c-A b l / F E 65$ nuclear translocation and activities inhibits APP-C99-induced neuronal cell death}

It had been reported that the nuclear function of $\mathrm{c}-\mathrm{Abl}$ is to regulate neuronal apoptosis. Therefore, we investigated 
(A)
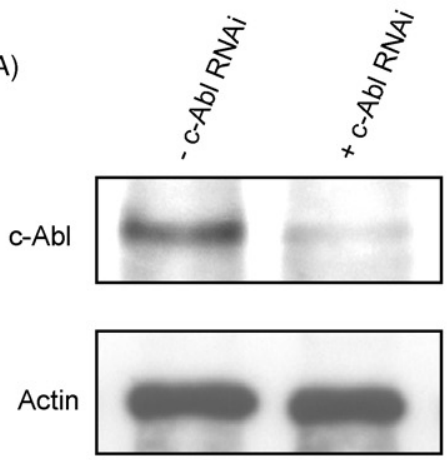

(C)

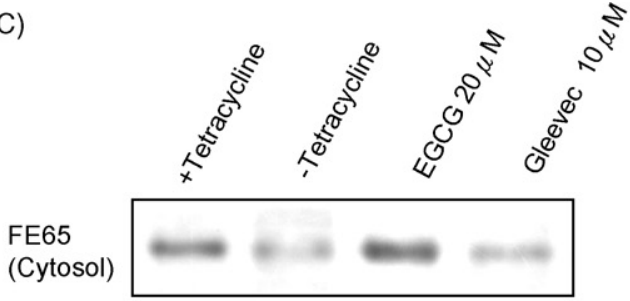

Actin

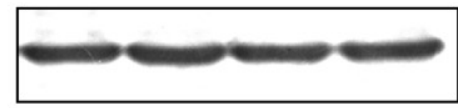

FE65 (Nucleus)

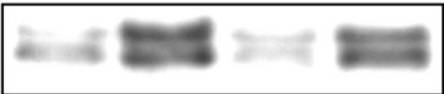

TFIIB

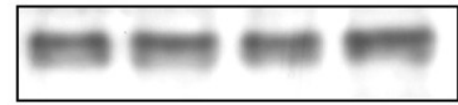

(D)
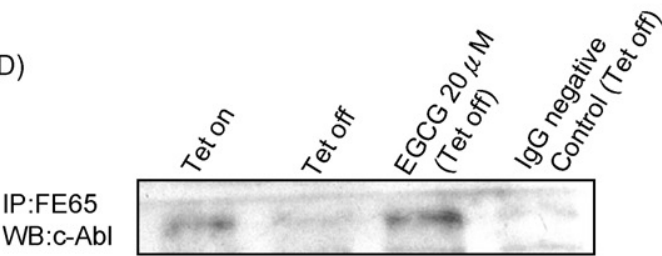

WB:c-Ab

(B)

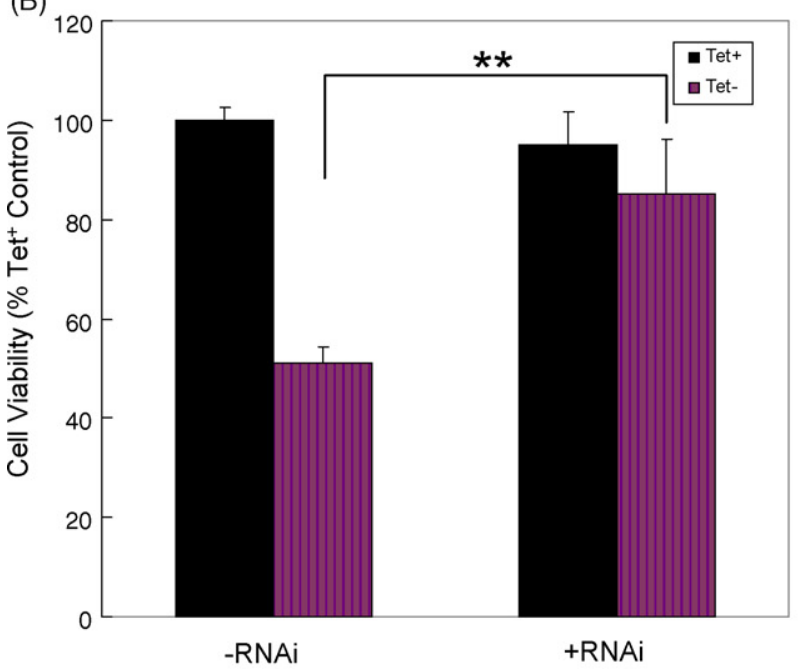

(E)

phosphoTyr ${ }^{216}-$ GSK3 $\beta$

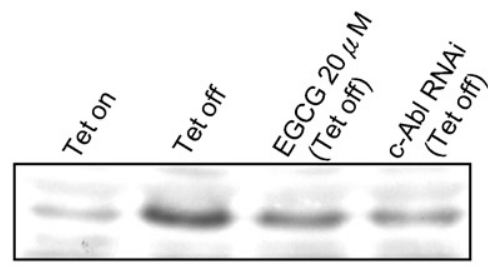

GSK3 $\beta$

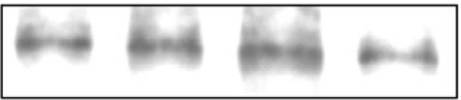

Actin

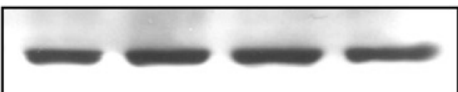

Fig. 4. Inhibition of c-Abl nuclear translocation and activities blocks APP-C99-induced neuronal cell death in MC65 cells. (A) MC65 cells transfected with c-Abl-RNAi interfered with c-Abl expression. (B) c-Abl-RNAi transfected MC65 cells could protect against APP-C99-induced neurotoxicity. RNAi transfected MC65 cells showed significant resistance to APP-C99-induced cell death after $18 \mathrm{~h}$. (C) Overexpression of APP-C99 in MC65 cells induced a significant increase in the levels of FE65 proteins in nuclear fractions for $72 \mathrm{~h}$. However, the EGCG treatment maintained FE65 subcellular distribution in the cytosolic fraction. (D) c-Abl-FE65 complex interaction could be decreased in response to the supplement of $20 \mu \mathrm{M}$ EGCG using the immunoprecipitation method. (E) APP-C99 overexpression increased GSK-3 $\beta$ phosphorylation at Tyr $^{216}$ for $72 \mathrm{~h}$, and both EGCG and c-Abl RNAi could block GSK-3 $\beta$ phosphorylation significantly. Bars depict mean \pm S.E. of at least three experiments. ' $* *$ ' represents a statistically significant difference non-tetracycline treated control group, $p<0.01$.

whether the c-Abl is important to APP-C99-induced neuronal cell death. We did it by using RNAi to inhibit c-Abl expression in MC65 cells. As shown in Fig. 4A, we transfected MC65 with c-Abl-RNAi to interfere with c-Abl protein expression. After $18 \mathrm{~h}$, the MC65 cells transfected with RNAi had reduced levels of $\mathrm{c}-\mathrm{Abl}$ compared to control neuronal cells. Once we induced APP-C99 overexpression by removing the medium tetracycline, the content of c-Abl was reduced, and transfected MC65 cells showed resistance to APP-C99- induced neurotoxicity (Fig. 4B). These results are consistent with previous data suggesting that $\mathrm{c}-\mathrm{Abl}$ participated in the APP-C99-induced neuronal cell death, revealing that the cAbl may play a key role in APP-C99-induced neurotoxicity.

To verify the mechanism at work in EGCG's blocking of the c-Abl nuclear translocation, we attempted to explore what factors might mediate c-Abl translocation in neuronal cells. It had been confirmed that FE65, an intracellular adaptor protein, could interact with c-Abl and stimulate its nuclear 
translocation (Perkinton et al., 2004). Mutation of the FE65 conserved domain inhibited binding of c-Abl and blocked APP-dependent GSK3 $\beta$ activation (Lau et al., 1999). To determine whether FE65 participated in these mechanisms, we evaluated the FE65 content by Western blotting. As shown in Fig. 4C, consistent with the interaction in a complex with c-Abl, overexpression of APP-C99 in MC65 cells induced a significant increase in the levels of FE65 proteins in nuclear fractions at $72 \mathrm{~h}$. However, the EGCG treatment but not Gleevec maintained FE65 subcellular distribution in the cytosolic but not the nuclear fraction. These data reveal EGCG may specifically inhibit c-Abl nuclear translocation by interrupting c-Abl-FE65 interaction. To validate this possibility, we analyzed the c-Abl-FE65 interaction by the protein immunoprecipitation method. In accordance with the above results, the c-Abl-FE65 complex showed a significant increase in response to the supplement of EGCG (Fig. 4D). Although GSK-3 $\beta$ is a constitutively active enzyme, its activity is associated with the phosphorylation status of the $\mathrm{Tyr}^{216}$ residue. Treatment with EGCG $(20 \mu \mathrm{M})$ resulted in a decrease in GSK-3 $\beta$ Tyr $^{216}$ phosphorylation compared to the APP-C99 overexpressed control group (Fig. 4E). In addition, pretreatment with c-Abl siRNA resulted in a decline of the basal level of $\mathrm{Tyr}^{216}$ phosphorylation, indicating that a c-Abl-dependent pathway is required for APP-C99-induced GSK-3 $\beta$ Tyr $^{216}$ phosphorylation.

\subsection{EGCG treatment reduces cerebral amyloidosis and c-Abl/FE65 interaction in Tg2576 transgenic mice}

The epidemiological evidence indicates a putative role of EGCG in the prevention and attenuation of neurodegenerative diseases. In addition, it has been reported that the orally administered EGCG can cross the blood-brain barrier and exert its bioactivities in mice (Suganuma et al., 1998). To verify whether EGCG treatment could prevent APP-induced neurodegeneration in vivo, we administered EGCG orally $(20 \mathrm{mg} / \mathrm{kg} /$ day) to $\mathrm{Tg} 2576$ transgenic mice as described in Section 2. As shown in Fig. 5A, A $\beta$ levels were markedly increased in the hippocampus of $\operatorname{Tg} 2576$ transgenic mice brains compared with wild-type mice. Accordingly, both mice hippocampal $A \beta_{1-40}$ and $A \beta_{1-42}$ levels were reduced by 21 and 27\%, respectively, after treatment with EGCG. The slice photomicrograph also showed representative $A \beta$ plaque pathology in the hippocampus, and EGCG significantly decreased the number of $A \beta$ plaques (data not shown). These data suggest that EGCG may act as an inhibitor in the transgenic mice model of APP-generated $A \beta$ and partially decrease the $A \beta$ levels to those of $\mathrm{Tg} 2576$ mice.

In concert with reduced c-Abl-FE65 interaction in cell culture data, EGCG treatment results showed a decreased c-Abl-FE65 interaction in tissue lysates (Fig. 5B). GSK3 $\beta$ has been recently proposed as downstream of c-Abl promoting neuron apoptosis in AD (Takashima, 2006). Therefore, we evaluated the effect of GSK3 $\beta$ inhibition by EGCG on hippocampal neuronal cell death induced by APP overexpres- sion. To evaluate the GSK3 $\beta$ inhibition by EGCG treatment, we analyzed GSK $3 \beta$ activities by monitoring its $\mathrm{Tyr}^{216}$ phosphorylation. As illustrated, our data show that overexpression of APP elevated GSK3 $\beta$ phosphorylation while the presence of EGCG significantly diminished it (Fig. 5C). We also measured escape latency by using the Morris water maze task, and the result indicated that mice with impaired spatial memories had significantly improved scores while receiving long term EGCG, indicating that the memory deficits in Tg2576 mice were reversed and memory was restored by EGCG treatment (data not shown). Taken together, these data suggest that the reduction of both APP-generated A $\beta$ and c-Abl/FE65 interaction in response to EGCG treatment is likely responsible for decreasing the retention of spatial memory in Tg2576 transgenic mice.

\section{Discussion}

Previous reports have shown that green tea components such as EGCG have significant neuroprotective properties; however, a clear cellular molecular mechanism underlying these effects has not been established. In this study, our concordant findings from MC65 cultured neuronal cells and $\operatorname{Tg} 2576$ transgenic mice raise the possibility of a suppressing effect by EGCG upon $\beta$-amyloid plaque deposition and neurotoxicity. Since the $A \beta$ levels were reduced both in vitro and in vivo, it is likely that the effect of EGCG is to prompt APP processing, as is consistent with previous reports (Rezai-Zadeh et al., 2005). However, the result that followed exposure to exogenous $\mathrm{A} \beta$ showing significant neuroprotective effects in SK-N-MC cells, combined with the reverse effect following EGCG treatment, suggests that EGCG may exert its protective effects through some mechanisms other than promoting APP nonamyloidogenic proteolysis.

In our data, EGCG has significant inhibitory effects on $\mathrm{c}-\mathrm{Abl}$ nuclear distribution. It has been pointed out that $\mathrm{A} \beta$ neurotoxicity is associated with an increase in c-Abl activity by increasing ROS production and that nuclear c$\mathrm{Abl}$ is responsible for neuronal apoptosis (Alvarez et al., 2004). This indicates that oxidative stress and $\mathrm{c}-\mathrm{Abl}$ share a downstream mechanism with $A \beta$-induced neurotoxicity. Furthermore, it has been proposed that $A \beta$ activating to some neuronal targets leads to tau hyperphosphorylation (De Felice et al., in press). Additionally, antibodies against $A \beta$ peptides lead to a reduction of both GSK3 $\beta$ activation and tau phosphorylation (Ma et al., 2006), suggesting GSK3 $\beta$ is one of major downstream targets of $A \beta$ peptides. GSK3 $\beta$ is abundant in the CNS and phosphorylates tau at most sites hyperphosphorylated in neurofibrillary tangles, both in transfected cells (Hong et al., 1997; Lovestone et al., 1996) and in vivo (Munoz-Montano et al., 1997). This makes GSK3 $\beta$ one of the candidate kinases involved in the hyperphosphorylation of tau in AD. Our data showed that the GSK3 $\beta$ activation occurred through a c-Abl-dependent pathway, thus suggesting a putative link among $\mathrm{c}-\mathrm{Abl}$ nuclear translocation, 

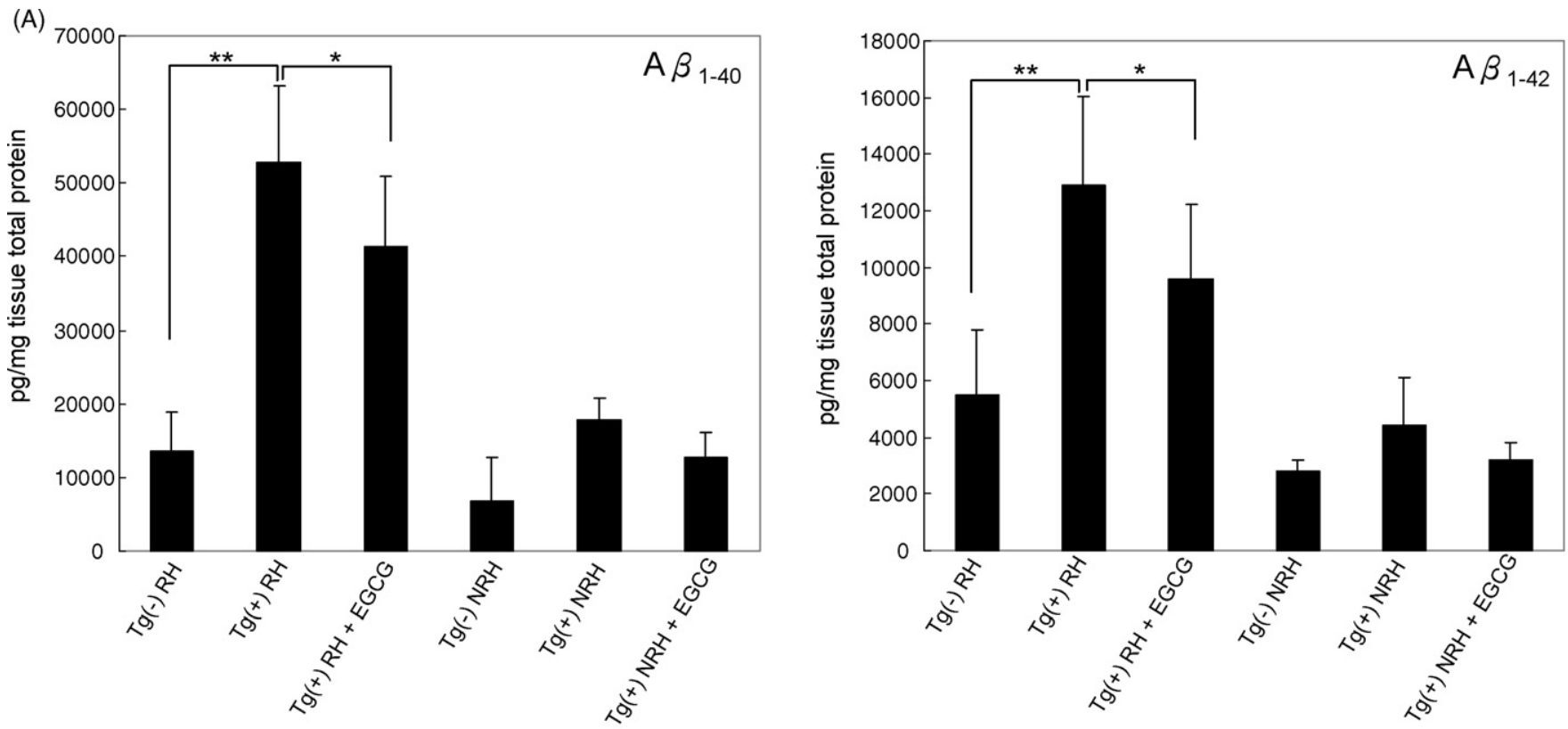

(B)

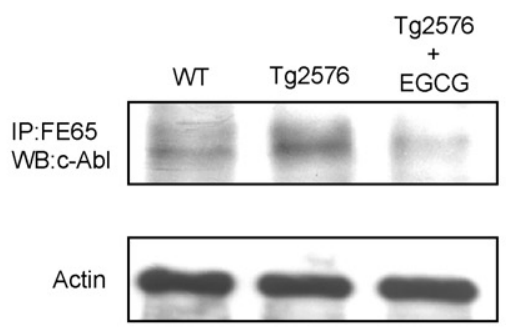

(C)
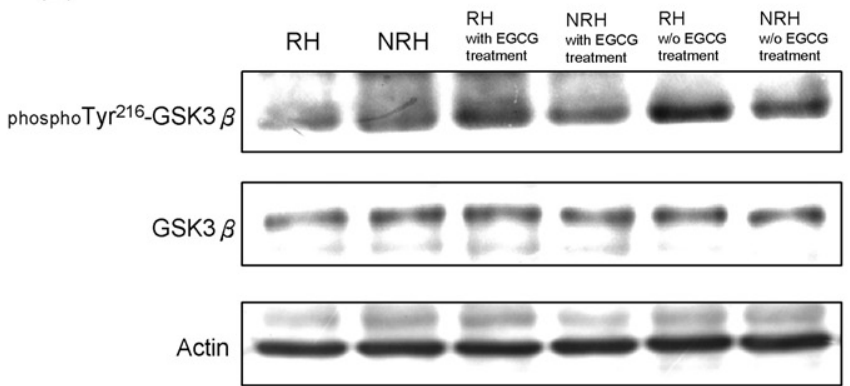

Fig. 5. Chronic EGCG treatment reduces cerebral $A \beta$ generation and c-Abl/FE65 interaction in Tg2576 transgenic mice. (A) Both $A \beta_{1-40}$ and $A \beta_{1-42}$ levels were markedly increased in hippocampus of Tg2576 transgenic mice brains treated with EGCG. After treatment with EGCG, both mice hippocampal A $\beta_{1-40}$ and $A \beta_{1-42}$ levels were reduced by 21 and 27\%, respectively. (B) EGCG treatment results showed a decreased c-Abl-FE65 complex interaction using the immunoprecipitation method in hippocampus of Tg2576 transgenic mice brains. (C) Overexpression of APP elevated the GSK $3 \beta$ Tyr ${ }^{216}$ phosphorylation, and the presence of EGCG significantly diminished the ability of the GSK3 $\beta \mathrm{Tyr}^{216}$ phosphorylation in hippocampus. H, hippocampus tissue; NH, non-hippocampus tissue; $\mathrm{R}$, right side; $\mathrm{Tg}(+), \operatorname{Tg} 2576$ transgenic mice; $\operatorname{Tg}(-)$, wild-type mice. Bars depict mean \pm S.E. of at least three experiments. '*' and '**' represent statistically significant differences from a wild-type control group, $p<0.05$ and $p<0.01$, respectively.

GSK3 $\beta$ activation, and tau phosphorylation in the pathogenesis of AD.

In addition, we have demonstrated that FE65 interacts with c-Abl followed by APP-C99 overexpression, which interaction can be inhibited by the treatment with EGCG. There is some evidence to support the idea that the FE65 serves in the regulation of cell survival may be directly related to the proteolytic processing of APP (Sabo et al., 1999). Furthermore, TACE, an ADAM family protease, cleaves APP at the cell surface in response to PKC activity, and this is also known to stimulate cell motility (Buxbaum et al., 1998; Obregon et al., 2006). These results are consistent with our notion that the regulation of c-Abl-FE65 interaction is the key agent of $A \beta$-induced neurotoxicity. Tyrosine phosphorylation of the C-terminal domain of APP, especially in $\mathrm{Thr}^{668}$, has been reported to show a regulation of FE65 nuclear localization (Perkinton et al., 2004). Once APP is phosphorylated, FE65 can be release from the cell membrane associated with c-Abl and then translocate into the nucleus. Thus, dephosphorylation of APP at Thr ${ }^{668}$ clearly decreases the $\mathrm{c}-\mathrm{Abl} / \mathrm{Fe} 65$ interactions and subsequently suppresses its nuclear signaling, such as GSK3 $\beta$ activation. In our preliminary data, EGCG was able to alter the neuronal membrane APP phosphorylation state and inhibit Thr ${ }^{668}$ phosphorylation (Supplemental Fig. 4). This supports the hypothesis that EGCG can function as a switch to control intracellular FE65 distribution, by preventing FE65 release from membrane APP and inhibiting c-Abl nuclear translocation.

In summary, the results reported here support recent findings indicating that treatment with EGCG enhances APP nonamyloidogenic proteolytic processing, but they also demonstrate that the EGCG-reduced nuclear translocation of 
c-Abl was independent of an influence on APP proteolytic processing. It appears that the neuroprotective action mechanisms of EGCG involve two steps: first, EGCG inhibits $\mathrm{A} \beta$ formation by inhibiting APP proteolytic processing, and secondly, EGCG inhibits cAbl/FE65 complex nuclear translocation and GSK3 $\beta$ activation. Additional studies will be needed to elucidate the relationships between the elevated markers of brain neurodegeneration, $\beta$-amyloid, and regulatory machinery of c-Abl.

\section{Disclosure statement}

No actual or potential conflict of interest. All animals were in compliance with protocols approved by the National Taiwan University Institutional Animal Care and Use Committee.

\section{Acknowledgements}

This work was supported by grants from the National Science Council, Taiwan (NSC94-2320-B002-118). Gleevec (Imatinib mesylate) was kindly provided by the Novartis Company. We thank Dr. George Martin and Dr. Qubai Hu, Department of Pathology, University of Washington for providing the MC65 cell line and excellent technical advice.

\section{Appendix A. Supplementary data}

Supplementary data associated with this article can be found, in the online version, at doi:10.1016/ j.neurobiolaging.2007.05.012.

\section{References}

Alvarez, A.R., Sandoval, P.C., Leal, N.R., Castro, P.U., Kosik, K.S., 2004. Activation of the neuronal c-Abl tyrosine kinase by amyloid-betapeptide and reactive oxygen species. Neurobiol. Dis. 17 (2), 326336.

Blass, J.P., 2002. Alzheimer's disease and Alzheimer's dementia: distinct but overlapping entities. Neurobiol. Aging 23 (6), 1077-1084.

Buxbaum, J.D., Liu, K.N., Luo, Y., Slack, J.L., Stocking, K.L., Peschon, J.J., Johnson, R.S., Castner, B.J., Cerretti, D.P., Black, R.A., 1998. Evidence that tumor necrosis factor alpha converting enzyme is involved in regulated alpha-secretase cleavage of the Alzheimer amyloid protein precursor. J. Biol. Chem. 273 (43), 27765-27767.

Choi, Y.T., Jung, C.H., Lee, S.R., Bae, J.H., Baek, W.K., Suh, M.H., Park, J., Park, C.W., Suh, S.I., 2001. The green tea polyphenol (-)epigallocatechin gallate attenuates beta-amyloid-induced neurotoxicity in cultured hippocampal neurons. Life Sci. 70 (5), 603-614.

Dai, Q., Borenstein, A.R., Wu, Y., Jackson, J.C., Larson, E.B., 2006. Fruit and vegetable juices and Alzheimer's disease: the Kame Project. Am. J. Med. 119 (9), 751-759.

De Felice, F.G., Wu, D., Lambert, M.P., Fernandez, S.J., Velasco, P.T., Lacor, P.N., Bigio, E.H., Jerecic, J., Acton, P.J., Shughrue, P.J., Chen-Dodson, E., Kinney, G.G., Klein, W.L. Alzheimer's disease-type neuronal tau hyperphosphorylation induced by Abeta oligomers. Neurobiol. Aging, doi:10.1016/j.neurobiolaging.2007.02.09, in press.

Drachman, D.A., 2006. Aging of the brain, entropy, and Alzheimer disease. Neurology 67 (8), 1340-1352.

Esposito, E., Rotilio, D., Di Matteo, V., Di Giulio, C., Cacchio, M., Algeri, S., 2002. A review of specific dietary antioxidants and the effects on biochemical mechanisms related to neurodegenerative processes. Neurobiol. Aging 23 (5), 719-735.

Floyd, R.A., Hensley, K., 2002. Oxidative stress in brain aging. Implications for therapeutics of neurodegenerative diseases. Neurobiol. Aging 23 (5), $795-807$.

Hardy, J.A., Higgins, G.A., 1992. Alzheimer's disease: the amyloid cascade hypothesis. Science 256 (5054), 184-185.

Hong, M., Chen, D.C., Klein, P.S., Lee, V.M., 1997. Lithium reduces tau phosphorylation by inhibition of glycogen synthase kinase-3. J. Biol. Chem. 272 (40), 25326-25332.

Hsiao, K., Chapman, P., Nilsen, S., Eckman, C., Harigaya, Y., Younkin, S., Yang, F., Cole, G., 1996. Correlative memory deficits, Abeta elevation, and amyloid plaques in transgenic mice. Science 274 (5284), 99-102.

Iqbal, K., Grundke-Iqbal, I., 2006. Discoveries of tau, abnormally hyperphosphorylated tau and others of neurofibrillary degeneration: a personal historical perspective. J. Alzheimers Dis. 9 (Suppl. 3), 219-242.

Lau, K.F., Miller, C.C., Anderton, B.H., Shaw, P.C., 1999. Molecular cloning and characterization of the human glycogen synthase kinase-3beta promoter. Genomics 60 (2), 121-128.

Levites, Y., Amit, T., Mandel, S., Youdim, M.B., 2003. Neuroprotection and neurorescue against Abeta toxicity and PKC-dependent release of nonamyloidogenic soluble precursor protein by green tea polyphenol (-)-epigallocatechin-3-gallate. FASEB J. 17 (8), 952-954.

Lovestone, S., Hartley, C.L., Pearce, J., Anderton, B.H., 1996. Phosphorylation of tau by glycogen synthase kinase- 3 beta in intact mammalian cells: the effects on the organization and stability of microtubules. Neuroscience 73 (4), 1145-1157.

Ma, Q.L., Lim, G.P., Harris-White, M.E., Yang, F., Ambegaokar, S.S., Ubeda, O.J., Glabe, C.G., Teter, B., Frautschy, S.A., Cole, G.M., 2006. Antibodies against beta-amyloid reduce Abeta oligomers, glycogen synthase kinase-3beta activation and tau phosphorylation in vivo and in vitro. J. Neurosci. Res. 83 (3), 374-384.

Mandel, S., Amit, T., Reznichenko, L., Weinreb, O., Youdim, M.B., 2006. Green tea catechins as brain-permeable, natural iron chelatorsantioxidants for the treatment of neurodegenerative disorders. Mol. Nutr. Food Res. 50 (2), 229-234.

Mandel, S., Reznichenko, L., Amit, T., Youdim, M.B., 2003. Green tea polyphenol (-)-epigallocatechin-3-gallate protects rat PC12 cells from apoptosis induced by serum withdrawal independent of P13-Akt pathway. Neurotox. Res. 5 (6), 419-424.

Miranda, S., Opazo, C., Larrondo, L.F., Munoz, F.J., Ruiz, F., Leighton, F., Inestrosa, N.C., 2000. The role of oxidative stress in the toxicity induced by amyloid beta-peptide in Alzheimer's disease. Prog. Neurobiol. 62 (6), 633-648.

Mosmann, T., 1983. Rapid colorimetric assay for cellular growth and survival: application to proliferation and cytotoxicity assays. J. Immunol. Methods 65 (1-2), 55-63.

Munoz-Montano, J.R., Moreno, F.J., Avila, J., az-Nido, J., 1997. Lithium inhibits Alzheimer's disease-like tau protein phosphorylation in neurons. FEBS Lett. 411 (23), 183-188.

Nakaya, T., Suzuki, T., 2006. Role of APP phosphorylation in FE65dependent gene transactivation mediated by AICD. Genes Cells 11 (6), 633-645.

Obregon, D.F., Rezai-Zadeh, K., Bai, Y., Sun, N., Hou, H., Ehrhart, J., Zeng, J., Mori, T., Arendash, G.W., Shytle, D., Town, T., Tan, J., 2006. ADAM10 activation is required for green tea (-)-epigallocatechin-3gallate-induced alpha-secretase cleavage of amyloid precursor protein. J. Biol. Chem. 281 (24), 16419-16427.

Perkinton, M.S., Standen, C.L., Lau, K.F., Kesavapany, S., Byers, H.L., Ward, M., McLoughlin, D.M., Miller, C.C., 2004. The c-Abl tyrosine kinase phosphorylates the Fe65 adaptor protein to stimulate 
Fe65/amyloid precursor protein nuclear signaling. J. Biol. Chem. 279 (21), 22084-22091.

Rezai-Zadeh, K., Shytle, D., Sun, N., Mori, T., Hou, H., Jeanniton, D., Ehrhart, J., Townsend, K., Zeng, J., Morgan, D., Hardy, J., Town, T., Tan, J., 2005. Green tea epigallocatechin-3-gallate (EGCG) modulates amyloid precursor protein cleavage and reduces cerebral amyloidosis in Alzheimer transgenic mice. J. Neurosci. 25 (38), 8807-8814.

Sabo, S.L., Lanier, L.M., Ikin, A.F., Khorkova, O., Sahasrabudhe, S., Greengard, P., Buxbaum, J.D., 1999. Regulation of beta-amyloid secretion by FE65, an amyloid protein precursor-binding protein. J. Biol. Chem. 274 (12), 7952-7957.

Selkoe, D.J., 2004. Alzheimer disease: mechanistic understanding predicts novel therapies. Ann. Intern. Med. 140 (8), 627-638.

Sinha, S., Lieberburg, I., 1999. Cellular mechanisms of beta-amyloid production and secretion. Proc. Natl. Acad. Sci. U.S.A. 96 (20), 11049 11053.
Smith, M.A., Perry, G., Richey, P.L., Sayre, L.M., Anderson, V.E., Beal, M.F., Kowall, N., 1996. Oxidative damage in Alzheimer's. Nature 382 (6587), 120-121.

Sopher, B.L., Fukuchi, K., Smith, A.C., Leppig, K.A., Furlong, C.E., Martin, G.M., 1994. Cytotoxicity mediated by conditional expression of a carboxyl-terminal derivative of the beta-amyloid precursor protein. Brain Res. Mol. Brain Res. 26 (1-2), 207-217.

Suganuma, M., Okabe, S., Oniyama, M., Tada, Y., Ito, H., Fujiki, H., 1998. Wide distribution of $[3 \mathrm{H}](-)$-epigallocatechin gallate, a cancer preventive tea polyphenol, in mouse tissue. Carcinogenesis 19 (10), 1771-1776.

Takashima, A., 2006. GSK-3 is essential in the pathogenesis of Alzheimer's disease. J. Alzheimers Dis. 9 (Suppl. 3), 309-317.

Van Etten, R.A., 1999. Cycling, stressed-out and nervous: cellular functions of c-Abl. Trends Cell Biol. 9 (5), 179-186.

Wang, J.Y., 2005. Nucleo-cytoplasmic communication in apoptotic response to genotoxic and inflammatory stress. Cell Res. 15 (1), 43-48. 